CHRISTIAN GRÜNNAGEL

ORCID: 0000-0003-0134-4684

Ruhr-Universität Bochum

christian.gruennagel@rub.de

\title{
LA POLITIQUE SELON LA MORT D'AGRIPPINE DE CYRANO DE BERGERAC : CONSPIRATION, ATHÉISME ET LIBERTINAGE ÉRUDIT SUR SCÈNE*
}

Si l'histoire littéraire se libérait enfin des préjugés d'un faux classicisme, elle mettrait ces deux ouvres [La Mort d'Agrippine et Le Pédant joué] parmi les plus grands du siècle ${ }^{1}$.

La Mort d'Agrippine, « a neglected play of strange and savage beauty $»^{2}$, dont la première eut probablement lieu en 1654, à la fin de la Fronde des princes, au théâtre renommé de l'Hôtel de Bourgogne ${ }^{3}$, est l'unique tragédie écrite par Cyrano de Bergerac et déclencha un véritable scandale littéraire à plusieurs échelles ${ }^{4}$. En ce

* Le présent article, issu d'un chapitre de ma thèse dédiée à la comparaison de la littérature (surtout dramatique) du XVII ${ }^{\mathrm{e}}$ siècle en France et en Espagne sous une perspective " baroque » (C. Grünnagel, Klassik und Barock - Pegasus und Chimäre. Französische und spanische Literatur des 17. Jahrhunderts im Dialog, Winter, Heidelberg 2010, pp. 146-161), a été mis à jour et adapté au sujet de ce volume de Romanica Wratislaviensia.

1 A. Adam, Histoire de la littérature française au XVII siècle, t. 2 : L’Époque de Pascal, Éditions Mondiales, Paris 1962, p. 123.

2 S. Read Baker, « Permutations of Parracide : Cyrano de Bergerac's La Mort d'Agrippine (1647)», The French Review 65.3, 1992, p. 375.

3 Voir C.J. Gossip, «Introduction », [dans :] Savinien Cyrano de Bergerac, La Mort d'Agrippine, C.J. Gossip (dir.), University of Exeter, Exeter 1983, p. VII $s q$.

${ }^{4}$ En ce qui concerne la première scandaleuse de la pièce, $c f$. M. Alcover, « Sisyphe au Parnasse : La réception des œuvres de Cyrano aux XVII ${ }^{\mathrm{e}}$ et XVIII ${ }^{\mathrm{e}}$ siècles », Euvres et Critiques 20.3, 1995, p. 230. 
qui concerne la forme, il semble bien qu'il s'agisse d'une tragédie régulière, donc classique $^{5}$, qui suive exactement le conseil horacien des cinq actes de même qu'elle respecte les trois unités aristotéliciennes. Le style est adéquat : ni mots bas ni mots sales ne s'y trouvent, et les bienséances sont (au moins apparemment) respectées ${ }^{6}$.

Toujours est-il que tromperie et trahison, dissimulation et mascarade ne sont pas uniquement les thèmes centraux de la pièce, mais on peut également se poser la question de savoir si cette tragédie n'intègre pas le « trompe-l'œil » au fond même de sa structure ${ }^{7}$. Le jeu avec le public commence dès le titre de la pièce, dont se dégagent deux problèmes : d'abord, la mort d'Agrippine est certes annoncée dans le titre, mais ne fait nullement partie de la tragédie : le trépas de la protagoniste ne fait même pas l'objet d'une allusion ${ }^{8}$. De plus, il paraît licite de se demander si la protagoniste féminine est bien choisie comme héroïne titulaire, alors qu'un autre personnage — Séjanus — reçoit un poids scénique au moins aussi important que la veuve impériale. Ce Séjanus est un affranchi devenu homme politique très important à la cour romaine de l'empereur Tibère. En même temps, Séjanus fait

5 «[C]lassic in form» (E.W. Lanius, Cyrano de Bergerac and the Universe of the Imagination, Droz, Genève 1967, p. 65). À comparer avec Jouslin qui trouve remarquable que Cyrano respecte les « emerging rules of French classical tragedy » (O. Jouslin, « Savinien de Cyrano de Bergerac », [dans :] Seventeenth-Century French Writers, F. Jaouën (dir.), Gale Group, Detroit-New York-San Diego 2003, p. 110).

6 D. Moncond'huy se prononce d'une façon sceptique par rapport à la bienséance de la pièce, mise en danger par des « violences » au niveau du langage scénique selon lui, mais aboutit, lui aussi, à la conclusion que « [1]a pièce est néanmoins conforme, dans une large mesure, à l'esthétique qui vient de s'imposer [...] » (idem, « Préface », [dans :] Cyrano de Bergerac, La Mort d'Agrippine. Veuve de Germanicus. Tragédie, édition établie et présentée par D. Moncond'huy, La Table Ronde, Paris 1995, p. $11 s q$ ). Toutes les citations de La Mort d'Agrippine renvoient à cette édition. Dans la suite, l'abréviation «MA » sera utilisée pour citer cette pièce cyranienne.

7 Voir J.-Ch. Darmon, Le songe libertin. Cyrano de Bergerac d'un monde à l'autre, Klincksieck, Paris 2004, p. 36 : « l'effet de trompe-l'œil qu'il [Cyrano] instaure d'emblée, le titre même [...] installe le spectateur dans cette logique des phasmata [.] ». Tant Gossip que Darmon soulignent l'importance centrale de la « feinte » dans la tragédie de Cyrano (voir C.J. Gossip, op. cit., p. XII, et J.-Ch. Darmon, « Entre le monde de La Mort d'Agrippine et L'Autre Monde des oiseaux-juges : variations lucrétiennes sur la mort et critique du politique selon Cyrano de Bergerac », Littératures classiques 55.3, 2005, p. 200 sq ; voir aussi O. Bloch, "Cyrano, Molière, et l'écriture libertine ", La Lettre Clandestine. Revue annuelle d'information sur la littérature philosophique clandestine de l'âge classique 5, 1996, p. $243 \mathrm{sq}$ : « toute la trame de [La Mort d'Agrippine] repose sur un véritable déchaînement de fourberie »).

8 S. Read Baker part de l'idée (erronée à mon avis) que les vers 1645-1646 annoncent la mort de l'héroïne titulaire (« D'elle et de Séjanus, les âmes déloyales / Arriveront ensemble aux plaines infernales » - S. Read Baker, op. cit., pp. 382 et 384, note 22). Dans cet aspect, je soutiens plutôt l'interprétation de D. Moncond'huy soulignant qu' « elle» (v. 1645) se réfère à Livilla (cf. MA, p. 125, note 12), ce qui est parfaitement en accord avec la logique du dénouement. Un autre argument est probablement encore plus pertinent et susceptible d'invalider l'opinion de Read Baker - au dernier acte, c'est Tibère lui-même qui se dirige vers Agrippine pour lui refuser la mort qu'elle attend : " Moi, te donner la mort ? J'admire ton audace ! / Depuis quand avec nous es-tu rentrée en grâce ? / Pour allonger tes maux, je te veux voir nourrir / Un trépas éternel dans la peur de mourir » (MA, vv. 1617-1620). 
partie des conspirateurs conjurés pour renverser l'empereur : quand le rideau se lève pour le premier acte, il a déjà formé une alliance avec deux conspiratrices, Livilla et Agrippine - une alliance instable, car tous les trois conspirent également entre eux et contre les autres participants de leur rébellion.

Séjanus n'est cependant pas qu'un simple conspirateur de plus autour de la prétendue héroïne titulaire de la tragédie : sa profession de « foi » comme athée et son courage inébranlable face à sa propre mort pendant la catastrophe de la pièce, son rôle de « soldat philosophe » (MA, v. 1650), disciple d'Épicure ${ }^{9}$, lui donnent une importance scénique centrale pour comprendre le contenu politique et philosophique de la seule tragédie cyranienne. Pour dégager cette importance de Séjanus pour une interprétation politique, il est nécessaire de jeter un coup d'œil analytique sur sa vision du monde et de l'État — une vision très proche des positions contemporaines qu'on désigne ordinairement comme libertinage érudit.

Homme issu de l'esclavage et dominé par son ambition effrénée, Séjanus est - comparable sur ce point au Richard III shakespearien - un des « monstres » théâtraux qui hantent les scènes européennes des $\mathrm{XVI}^{\mathrm{e}}$ et $\mathrm{XVII}^{\mathrm{e}}$ siècles ${ }^{10}$. Le scandale frappe déjà au second acte quand Séjanus émet devant son confident Térentius l'hypothèse que l'existence des dieux n'a pas d'autre fondement que la crédulité stupide et les illusions des hommes. Les cieux sont vides, les divinités de vains fantasmes qu'un esprit fort comme le sien n'a nul besoin de craindre, mais dont il peut profiter selon son calcul politique ${ }^{11}$ :

Térentius : Respecte et crains des dieux l'effroyable tonnerre.

Séjanus : Il ne tombe jamais en hiver sur la terre,

J'ai six mois pour le moins à me moquer des dieux,

Ensuite je ferai ma paix avec les Cieux.

Térentius : Ces dieux renverseront tout ce que tu proposes.

Séjanus : Un peu d'encens brûlé rajuste bien des choses.

(MA, vv. 629-634)

9 Pour Séjanus comme adepte d'Épicure, voir D. Moncond'huy, op. cit., p. 13. À compléter par le caveat prononcé par J.-Ch. Darmon par rapport à " l'épicurisme [...] dans La Mort d'Agrippine » (idem, "Libertinage et politique : remarques sur l'utilité et les incertitudes d'un questionnement », Littératures classiques 55.3, 2004, p. 19). Voir aussi son étude monographique (J.-Ch. Darmon, Le songe libertin..., Klincksieck, Paris 2004, pp. 34-36).

10 Pour la catégorie (esthétique) du monstre au XVII ${ }^{\mathrm{e}}$ siècle, voir C. Grünnagel, op. cit., chap. 3.4. En ce qui concerne la monstruosité de Richard III comme personnage dramatique, voir I.F. Moulton, « A Monster Great Deformed. The Unruly Masculinity of Richard III », Shakespeare Quarterly 47.3, 1996, pp. 251-268.

11 La position de Séjanus se trouve en accord avec les principes que le Père Garasse résume dans sa polémique contemporaine contre le libertinage érudit, car les « esprits forts » ne croyaient en Dieu que « par maxime d'Estat», selon lui (voir la maxime II dans : La Doctrine curieuse, [dans :] Les Libertins au XVII siècle, A. Adam (dir.), Buchet/Chastel, Paris 1964, p. 41). Pour une vision panoramique de la relation entre religion, politique et libertinage érudit, voir aussi J.-P. Cavaillé, «Imposture politique des religions et la sagesse libertine ", Littératures classiques 55.3, 2004, surtout p. 29 sq. 
Dans ce dialogue pointu, Séjanus représente un politicien machiavélique ${ }^{12}$ qui se base sur une vision scientifique du monde et qui sait donc très bien que les tonnerres ne dépendent pas d'une quelconque volonté divine, mais simplement du climat, c'est-à-dire d'un phénomène entièrement nature ${ }^{13}$. Il sait aussi qu'il sera amplement suffisant de jouer le dévot face au peuple ignorant en imitant les cérémonies religieuses ${ }^{14}$. L' « encens » mentionné par Séjanus ajoute une allusion risquée aux pratiques de l'Église catholique de l'époque, qui se trouvent mises en parallèle (dangereux) avec le polythéisme païen de la Rome impériale. Face à la critique de la religion exposée par Séjanus, son conseiller Térentius représente un croyant pour qui l'utilité pratique de la religion « ici-bas » s'allie naturellement à ses convictions théologiques. Même pour le plan de terrasser l'empereur, la religion pourrait s'avérer avantageuse puisque, selon Térentius, les dieux nous donnent le courage de vaincre notre crainte de la mort — une crainte évidemment très présente car la punition pour conspiration contre l'empereur est la peine capitale. Pour se fortifier contre cette crainte, Térentius recommande la foi : « Qui les craint [les dieux], ne craint rien » (MA, v. 635) - un argument vite détourné contre le croyant par Séjanus qui professe sa conviction athée face à son confident, mais également au public très majoritairement chrétien de l'époque :

[...] Ces enfants de l'effroi,

Ces beaux riens qu'on adore, et sans savoir pourquoi,

Ces altérés du sang des bêtes qu'on assomme,

Ces Dieux que l'homme a faits, et qui n'ont point fait l'homme,

Des plus fermes États ce fantasque soutien,

$\mathrm{Va}$, va, Térentius, qui les craint, ne craint rien.

$$
\text { (MA, vv. 635-640) })^{15}
$$

12 Voir E. Harth, Cyrano de Bergerac and the Polemics of Modernity, Columbia University Press, New York-London 1970, p. 25 sq. La réception critique de Machiavel comme « atheorum [...] princeps » prend son essor avec les écrits de Vanini (voir J.-Ch. Darmon, « Entre le monde de La Mort d'Agrippine... », p. 222).

13 Voir aussi J.-Ch. Darmon, Le songe libertin..., p. 30.

14 L'utilité de la religion à des fins complètement étrangères au salut de l'âme de l'individu est un sujet traité plusieurs fois dans des écrits et sur les scènes du XVII ${ }^{\mathrm{e}}$ siècle ; voir, par exemple, le Tartuffe ou le Dom Juan de Molière, et surtout La Bruyère critiquant les « Esprits forts » pour « faire servir Dieu et la religion à la politique » (Les Caractères, XVI, 3, E. Bury (dir.), Le Livre de poche, Paris 1995, p. 574; voir aussi le commentaire de J.-Ch. Darmon, « Libertinage et politique... », p. $17 s q$ ). Concernant le machiavélisme de Séjanus qui culmine dans une vision de la religion comme « imposture à l'usage des politiques », voir J.-Ch. Darmon, « Entre le monde de La Mort d'Agrippine... », pp. 213-215. La stratégie de Séjanus de « rajuster bien des choses » en se pliant aux conventions religieuses coram publico est aussi en accord avec d'autres écrits libertins de l'époque. Voir, par exemple, les Lettres de Guy Pantin : «Si dans l'exercice externe de la Religion quelque chose vous desplaist, n'en dites mot, cachez vostre maltalent et n'en parlez point. Croyez-ens ce que vous devez, et laissez-là le reste sans causer aucun scandale. Intus ut libet, foris ut moris est, pratiquez ce bon mot des Italiens » (dans : J.-Ch. Darmon, « Libertinage et politique... », p. 13 sq, note 12).

15 La position de Séjanus annonce (avant la lettre) la critique de la religion au XIX ${ }^{\mathrm{e}}$ siècle, à commencer par la perception du Créateur comme création de l'homme qui nous renvoie aux 
Séjanus pervertit la sémantique de l'énoncé original que Térentius vient de prononcer, sans absolument rien changer par rapport au signifiant ${ }^{16}$ : celui qui craint les dieux n'a rien à craindre sur terre, disait Térentius ; son maître lui réplique : celui qui craint les dieux, s'effraye d'une chimère car les dieux n'existent pas $!{ }^{17}$ La signification ontologique du « rien » comme néant est habilement soulignée par la structure du vers et la rime employée : tandis que le «rien » de Térentius se trouve juste avant la césure de l'alexandrin et avant de passer la parole à Séjanus, celui-ci place son propre « rien» à la cadence du vers, renforcée encore par la rime (MA, v. 639) et la réinterprétation de la phrase initiale : « [q]ui les craint, ne craint rien » $(\mathrm{MA}, \mathrm{v} .635)^{18}$.

Cette provocation athée fut à l'origine du scandale causé par l'œuvre ${ }^{19}$ et y culmine dans une sorte d'argument cynique sur la non-existence des dieux : Séjanus détruit d'abord l'argument (traditionnel) physico-théologique censé prouver l'existence nécessaire d'une divinité créatrice d'une façon extrêmement arrogante (« Superbe ! ${ }^{20}[\mathrm{MA}$, v. 577$\left.]\right)$, pour présenter ensuite sa propre existence criminelle comme preuve de l'absence radicale d'une quelconque justice divine et, par conséquent, des dieux eux-mêmes ${ }^{21}$ :

Térentius : Mais s'il n'en était point [de dieux], cette machine ronde... ?

Séjanus : Oui, mais s'il en était, serais-je encore au monde?

(MA, vv. 641-642)

Il faut certes souligner que l'argument introduit par Séjanus se réfère aux dieux païens dont l'Église aussi nie l'existence ${ }^{22}$; les contemporains de Cyrano n'étaient pourtant pas dupes de ce camouflage antiquisant : le scandale provoqué par sa tragédie montre qu'on comprenait clairement que les vers de Séjanus visaient également le dieu chrétien et tous les systèmes théologiques basés sur le concept du théisme.

thèses de Ludwig Feuerbach (Das Wesen des Christentums [L'Essence du christianisme], Leipzig 1841).

16 Selon J.-Ch. Darmon, ce « jeu sur les signifiants » est un trait caractéristique général de la rhétorique employée par Séjanus (« Entre le monde de La Mort d’Agrippine... », p. 210). Voir aussi J.-Ch. Darmon, Le songe libertin..., p. 36 sq.

17 Voir aussi le commentaire d'André Blanc (S. Cyrano de Bergerac, Euvres complètes, t. 3, A. Blanc (dir.), Honoré Champion, Paris 2001, p. 298, note 161).

18 J.-Ch. Darmon classifie cette figure comme « pointe par équivoque » et la range parmi les jeux langagiers du libertinage érudit en nous renvoyant au cas de Théophile de Viau («Entre le monde de La Mort d'Agrippine... », p. 202).

19 Voir ibidem, p. $190 s q$, et H. Mazaheri, « La Conception matérialiste de la mort dans La Mort d'Agrippine de Cyrano de Bergerac », Cahiers du dix-septième 6, 1992, p. 55.

${ }^{20}$ Voir l'étymologie latine (superbus) qui évoque le péché mortel de la superbia dans le christianisme. Agrippine se voit tâchée de la même épithète (" superbe ») dans la bouche de Tibère (cf. MA, v. 1641).

${ }^{21}$ Voir aussi J.-Ch. Darmon, Le songe libertin..., p. 30 sq.

22 Voir aussi J.-P. Cavaillé, « Histoire d'équivoques », Cahiers du Centre de recherches historiques 33, avril 2004, p. 165. 
L'existence de Dieu démasquée, donc, comme illusion humaine ? L'ordre du monde et de l'État équivalant à l'usurpation et à la tyrannie, comme nous le verrons ensuite ? La résistance contre le tyran - un crime ? Le dialogue entre hommes - un trompe-l'œil ?23 Il devrait être aisé de voir que cette tragédie politique et philosophique avait bel et bien le pouvoir de troubler profondément les contemporains de Cyrano. De même, il n'est pas surprenant que les représentations de La Mort d'Agrippine fussent supprimées après la première.

Comme il est indispensable de se concentrer sur quelques exemples qui nous montrent la perversion ${ }^{24} \mathrm{du}$ discours — et surtout du discours politique ! — dans La Mort d'Agrippine, il paraît souhaitable de suivre d'abord, encore plus, le dialogue de Séjanus et de son confident Térentius de l'acte second, un dialogue qui se termine par la profession de « foi » athée prononcée par l'un des principaux conspirateurs de la pièce.

Térentius a d'abord critiqué son maître comme arrogant, " superbe », donc proche de l'hubris antique, puisque Séjanus base son ambition démesurée sur sa filiation sans aucun capital ni économique ni symbolique : ce qu'il veut, c'est s'élever d'« homme de rien » (MA, v. 587) pour devenir empereur, maître de l'Empire romain ; et puisque l'empereur actuel Tibère est l'obstacle principal à son plan, il est nécessaire de se débarrasser de lui. Comme confident, Térentius occupe la place d'une sorte de conscience morale extériorisée de Séjanus dans ce dialogue : « Mais le crime est affreux de massacrer son maître! » (MA, v. 597). La réponse du conspirateur affranchi qui aspire au trône des césars est digne d'être citée plus extensivement, car elle nous présente de manière exemplaire un des lieux rhétoriques centraux de la pièce — le monde renversé comme allégorie politique :

Mais on devient au moins un magnifique traître.

Quel plaisir sous ses pieds de tenir aux abois

Celui qui sous les siens fait gémir tant de Rois !

Fouler impunément des têtes couronnées,

Faire du genre humain toutes les destinées,

Mettre aux fers un César, et penser dans son cœur :

«Cet esclave jadis était mon Empereur».

$$
\text { (MA, vv. 598-604) }
$$

Térentius lui rappelle les risques énormes de cette entreprise criminelle :

${ }^{23}$ La figure discursive du trompe-l'œil est tellement fréquente et complexe dans la pièce de Cyrano que l'editio princeps de 1654 introduit même un guide de lecture in nuce à deux reprises pour orienter les lecteurs : cf. MA, vv. 895-898 («Vers équivoques») et vv. 1229-1234 ("Vers qui cachent un autre sens »), quelque chose d'exceptionnel, à mon savoir, dans la publication d'un ouvrage contemporain au Grand Siècle.

24 Dans ma thèse, j'ai proposé le terme «perversion» (sous la forme quelque peu transfigurée de «Per=version », introduite pour ne pas évoquer uniquement la sémantique négative du mot) comme possibilité terminologique capable de rassembler sous un seul terme plusieurs caractéristiques de l'époque tant au niveau de l'histoire — de la fable dans le cas du drame — qu'au niveau du discours (rhétorique) d'œuvres littéraires (voir C. Grünnagel, op. cit., chap. 3.3). 
Térentius : Peut-être en l'abattant tomberas-tu toi-même ?

Séjanus : Pourvu que je l'entraîne avec son diadème,

Je mourrai satisfait, me voyant terrassé

Sous le pompeux débris d'un trône renversé.

(MA, vv. 605-608)

Le monde renversé, « perversion » de l'ordre (politique et social), est introduit à travers des images troublantes : l'empereur enchaîné, l'empereur esclave de son esclave, qui se place lui-même sur un " trône renversé ». Il est cependant important de souligner que ce mundus inversus ne constitue point le renversement d'un «bon ordre établi $»^{25}$, selon la définition que donnent les dictionnaires de l'époque du verbe " pervertir ", mais se situe dans la logique d'un monde politique basé entièrement sur le crime, la trahison et le meurtre. Ce que des figures rhétoriques introduisent comme simple lieu commun, comme monde renversé topique, s'avère donc beaucoup plus complexe au niveau de la trame dramatique, car la conspiration de Séjanus, son plan de renverser l'empereur, suit exactement la logique de ce monde perverti qui aurait pris son départ avec l'assassinat de Germanicus, feu époux de l'héroïne titulaire, Agrippine. Pour elle, le responsable de ce crime « originel » est avant tout Tibère dont la veuve veut se venger à tout prix.

Dans le contexte baroque de la dialectique de l'être et du paraître, de la dissimulation et de la tromperie, de soi-même autant que les autres, la confrontation d'Agrippine et de l'empereur Tibère au deuxième acte nous livre un bon exemple de cette rhétorique trompeuse qui caractérise le langage poétique de la tragédie cyranienne et se prête à une interprétation politique ${ }^{26}$. La seconde scène du second acte réunit tous les personnages principaux, sauf Livilla, l'autre conspiratrice : y sont présents l'empereur et ses ennemis conjurés, Séjanus et Agrippine, tous avec leurs confidents, à savoir Nerva pour l'empereur et Térentius pour Séjanus, avec la notable exception que Cornélie, la confidente d'Agrippine, manque à cet entretien. Le polylogue est comparable à un " concours » d'art de la tromperie grâce à un emploi linguistique artificieux, un « concours » annoncé au début par Tibère luimême quand il aperçoit Agrippine venant vers lui :

Mais la [Agrippine] voici... N'importe il faut la régaler

D'une offre dont l'éclat suffit pour l'aveugler.

Vois comme son front cache et montre sa vengeance,

Et dans quelle fierté la superbe s'avance!

Pour me tromper encore elle vient en ces lieux ;

Mais écoute-nous feindre à qui feindra le mieux.

(MA, vv. 427-432 [je souligne])

${ }^{25}$ Dictionnaire de l'Académie Française, t. 2, Librairie Arthème Fayard / Imprimerie nationale, Paris 1968, s. v. : pervertir (je souligne).

26 J.-Ch. Darmon souligne l'hypertrophie du trompe-l'œil dans La Mort d'Agrippine (« une telle densité d'effets de tromperie ») et présente une contextualisation de ce phénomène dans le discours politique de l'époque (voir J.-Ch. Darmon, « Rhétorique du songe, fictions heuristiques et politiques de la "grimace" : Cyrano sur les traces de Quevedo, de Kepler et de Campanella », Littératures classiques 53, 2004, p. 196). 
Nous voilà maintenant au cœur même de la rhétorique politique de Rome comme Cyrano la conçoit sur scène - des discours affreux qui constituent aussi le passe-temps de ceux qui sont censés garantir la justice, la vérité et l'ordre. La rhétorique baroque à l'œuvre dans la tragédie de Cyrano est vouée entièrement à une esthétique de tromperie et d'illusion ${ }^{27}$. Les acteurs et leurs stratégies linguistiques ressemblent aux tricheurs d'une partie de cartes qui permettraient au public de jeter des coups d'œil sur leur jeu grâce à une technique dramatique consistant à se tourner fréquemment vers leurs confidents en aparté. Comme Tibère et Agrippine sont deux maîtres du discours politico-machiavélique, il n'est pas facile de dire qui est vainqueur ou vaincu dans ce jeu langagier et dangereux — car il y va de la vie. Tandis qu'Agrippine « rassure » l'empereur de pouvoir compter sur le soutien des troupes, complices de la veuve de Germanicus ( $\ll$ J'amène à ton secours mes proches, mes amis » [MA, v. 439]), Tibère nous montre de son côté qu'il a très bien compris que ces « amis » ne sont autres que ses bourreaux in spe et inverse les mots empoisonnés pour les retourner à la destinatrice : il aurait déjà pris des mesures pour « remercier » Agrippine pour ce « soutien » en déployant ses légions autour de Rome - une mesure prise par le seul souci de protéger Agrippine et le fils de Germanicus de l'hostilité de la populace de Rome. Tibère va si loin dans son discours de feinte qu'il prétend vouloir installer le fils de son adversaire sur son propre trône, par les armes de ses légions stationnées près de la métropole :

[...] Généreuse Princesse,

Je ne puis par ma bouche exprimer ma tendresse :

Car un moindre présent que le trône d'un roi

Ne saurait m'acquitter de ce que je te dois ;

De Rome à ce dessein j'approche mon armée,

Pour forcer cette esclave au joug accoutumée

D'adorer dans ton fils ce prince bien-aimé,

L'image d'un héros qu'elle a tant estimé [Germanicus] :

Oui, je viens sur son front déposer ma couronne,

Et quiconque osera choquer ce que j'ordonne,

C'est un traître, un mutin, qu'en vassal plein de cœur

J'immolerai moi-même au nouvel empereur.

(MA,vv. 441-452)

Agrippine n'est quand même pas dupe car elle voit très bien que l'offre de Tibère n'est qu'un piège : le dessein énoncé d'abdiquer et de céder le trône au fils de Germanicus est complètement entouré de menaces à peine camouflées : dans le discours tibérien, le «nouvel empereur » est délibérément placé à l'extrême limite du vers, seul, à la fin de la tirade, tandis que l'armée de Tibère domine le champ dès le vers 445, soumet Rome (allégorisée) et implicitement aussi Agrippine

27 La composition rhétorique de la tragédie s'intègre donc d'une façon parfaite dans le contexte de la théorie politique des penseurs libertins de l'époque comme, par exemple, l'auteur anonyme du Theophrastus redivivus (voir J.-P. Cavaillé, «Imposture politique des religions... », p. 30 : « le corps politique apparaît comme le règne de l'imposture et du mensonge »). 
comme « esclave ». L'empereur actuel souligne clairement qu'il n'abandonnera jamais le pouvoir et qu'il sait très bien punir la trahison et la mutinerie (cf. MA, v. 451) - c'est-à-dire tout ce qu'Agrippine projette.

La réaction d'Agrippine essaye de renverser encore une fois la sémantique alambiquée du discours impérial à l'aide d'antithèses et de paradoxes qui nous renvoient à la figure déjà analysée du mundus inversus. Le verbe estimer y prend un sens particulièrement amer dans les bouches d'une conspiratrice et d'un despote qui ne ressentent que de la haine pour l'adversaire politique :

Qui renonce à sa gloire en offrant sa couronne,

Il en acquiert, César, plus qu'il n'en abandonne ;

Tu m'estimes beaucoup de me la présenter,

Mais je m'estime trop pour pouvoir l'accepter;

C'est en la refusant qu'on s'en doit rendre digne,

Je veux que l'univers en juge par ce signe.

(MA, vv. 453-458 [je souligne])

En luttant pour la couronne impériale, les deux ennemis échangent encore d'autres arguments, pointus comme des coups dans un duel à l'épée 28 - un dialogue qui culmine dans le renversement de toute une série d'impératifs déployés en un parallélisme syntaxique parfait :

Tibère : Règne, je te l'ordonne, et régnant fais connaître

Que tu sais m'obéir encor comme à ton maître.

Agrippine : Règne, je te l'ordonne, et respectant ma loi,

Obéis pour montrer que tu n'es plus mon roi ;

Règne et puisque tu veux me rendre souveraine,

Montre en m'obéissant que je suis déjà reine.

(MA, vv. 505-510)

Dans sa totalité, ce duel rhétorique est d'une très grande complexité. Il s'avère surtout difficile de comprendre pourquoi Agrippine rejette catégoriquement l'offre de Tibère. Le public pourrait se demander pourquoi elle ne feint pas d'accepter la couronne pour son fils dans l'espoir d'utiliser le pouvoir nouvellement acquis pour se venger de Tibère. Il est clair que nous, spectateurs ou lecteurs, nous sentons incapables de discerner des raisons exactes susceptibles d'expliquer le comportement d'Agrippine ${ }^{29}$. La raison de cette obscurité rhétorique s'explique quand même assez bien : la veuve de Germanicus fait face au public comme une tricheuse qui

${ }^{28}$ La confrontation de Tibère et d'Agrippine à l'acte IV suit aussi ce modèle d'un « duel » rhétorique. Là, l'empereur invite expressément son adversaire à prêter plus d'attention à ses parades : « Défends-toi, défends-toi mieux » (MA, v. 1037).

29 À comparer avec la préface de D. Moncond'huy : «Une telle pratique du mensonge déconcerte immanquablement le spectateur, habitué à croire les personnages. Il faut bien un ou deux actes pour qu'on comprenne que les règles du jeu ne sont pas les mêmes que dans une autre pièce $[\ldots]$ » (op. cit., p. $15 s q$ ). 
protège son jeu des regards ${ }^{30}$, parce qu'elle est la seule sur scène à être sans confident(e). Alors que Nerva exerce cette fonction pour Tibère, Cornélie, la confidente d'Agrippine, est absente. Le spectateur n'a donc d'autre possibilité d'interpréter les motivations d'Agrippine qu'en pesant ses arguments prononcés expressis verbis sur scène. Comme les autres personnages de la pièce — l'empereur et Séjanus —, nous dépendons complètement de la surface de son discours, tandis que les remarques en aparté des hommes politiques nous apportent des informations pour juger de leurs subterfuges. Dans la scène suivante, Séjanus se fait écho de cette sensation troublante qu'Agrippine n'a pas seulement trompé Tibère, mais aussi le public « témoin » du dialogue :

Lorsque contre soi-même avec nous il conspire,

Quelle raison vous meut à refuser l'empire?

(MA, vv. 517-518)

Qui ou quelle raison a donc empêché Agrippine d'accepter l'offre de l'empereur, qui a proposé quelque chose de paradoxal comme s'il était un conspirateur de plus, conspirant contre lui-même, dans une Rome où " empire » rime toujours avec la forme verbale " conspire » ? La veuve de Germanicus y répond d'abord par un exposé rationnel de ses motifs, pour expliquer ensuite ses actes sur une base plutôt irrationnelle, une explication en cohérence avec le caractère du personnage construit par Cyrano, mais impossible à dégager des mots qu'elle a prononcés face à Tibère : oui, elle aurait vu clairement que l'offre de Tibère était une feinte et un guet-apens. Elle connaitrait parfaitement l'homme suivant toutes les maximes du libertinage érudit - pensons à Descartes : larvatus prodeo - et saurait discerner être et paraître dans son discours pour démasquer les véritables motifs de l'empereur :

Il était trop masqué pour être reconnu ;

Un homme franc, ouvert, sans haine, sans colère,

Incapable de peur, ce n'est point là Tibère ;

Dans tout ce qu'il paraît, Tibère n'est point là :

Mais Tibère est caché derrière tout cela.

(MA, vv. 524-528 [je souligne])

Tibère aurait feint la générosité pour mettre à l'épreuve les desseins d'Agrippine, pour voir si elle aspirait vraiment à le renverser ${ }^{31}$, et il aurait alors pris des mesures fatales si elle avait accepté son offre trompeuse.

Voilà la partie encore raisonnable de la réponse qu'Agrippine donne à Séjanus, une explication qui souligne la cohérence de notre propre interprétation de son discours. Un autre aspect s'y ajoute cependant et s'avère beaucoup plus important que ces raisons : Agrippine est entièrement dominée par la volonté de se venger

${ }^{30}$ Nous trouvons le motif du tricheur également dans la peinture de l'époque, surtout chez Caravaggio et Régnier (voir H. Langdon, " Falschspieler, Zigeuner und Hausierer », [dans :] Die Geburt des Barock, B.L. Brown (dir.), Belser, Stuttgart 2001, pp. 44-55).

31 À comparer avec MA, vv. 529-530, et E.W. Lanius, op. cit., p. 77. 
de Tibère qu'elle perçoit comme un monstre politique ${ }^{32}$. C'est pourquoi elle sent la nécessité de réduire l'empereur à n'être plus qu'un tyran abject. Si elle avait accepté son offre, Tibère y aurait gagné au moins une apparence de générosité - ce qui aurait été inacceptable pour Agrippine. Sa couronne n'est donc rien pour elle face à sa vengeance, qui préside à toutes ses actions. Par conséquent, Agrippine ne veut pas se contenter de la perspective trompeuse de voir son fils empereur :

Sache que je préfère à l'or d'une couronne

Le plaisir furieux que la vengeance donne ;

(MA, vv. 537-538)

Et je veux que le rang qu'il [Tibère] me retient à tort

Me conserve toujours un motif pour sa mort.

(MA, 547-548)

L'obsession d'Agrippine, c'est la vengeance. Son but de punir Tibère pour l'assassinat de Germanicus est, hélas, incompatible avec l'ambition de Séjanus avec qui elle feint d'être conjurée contre l'empereur en feignant également d'aimer l'ancien esclave, maintenant homme politique et conspirateur dans la Rome impériale. Dévoré par son orgueil et par son ambition de se placer sur le trône des césars où Tibère essaye de se maintenir coûte que coûte, Séjanus n'est plus qu'un instrument de la vengeance d'Agrippine.

Tous les personnages de la tragédie de Cyrano nous apparaissent donc comme si le malin génie de Descartes (le genius malignus) ${ }^{33}$ les avait placés dans un monde où tout n'est que tromperie macabre, où il n'est possible de se fier ni à une divinité — car les cieux sont vides, selon Séjanus — , ni aux mots des « amis », des « confidents ». L'ordre du monde est perverti. À la tête de l'État se trouve Tibère, homme politique machiavélique, voire assassin, comme arbiter orbis, source et garant de la loi. L'empereur n'est pas seulement pour Agrippine, mais pour le public aussi, une monstruosité politique ; ses ennemis, les conjurés, ne sont, hélas, nullement préférables $^{34}$. La configuration de tous ces personnages est proche d'une parodie

${ }^{32}$ La figure du monstrueux se trouve également dans la tragédie de Cyrano ( $c f$. MA, vv. 11131114 et v. 1405 visant Tibère ; v. 1170 : dans le style du Typhon, épopée burlesque de Scarron). Pour cette figure discursive au XVII ${ }^{\mathrm{e}}$ siècle, voir C. Grünnagel, op. cit., chap. 3.4. ; pour une appréciation du Typhon de 1648, donc contemporain de Cyrano : ibidem, pp. 90-93.

33 Les Méditations de Descartes étaient déjà publiées en 1641. Comme nous savons que Cyrano connaissait la physique cartésienne et employait sa terminologie (par exemple, distinguer clairement) dans ses œuvres (voir M. Alcover, Cyrano relu et corrigé, Droz, Genève 1990, pp. 117-167, chap. « Cyrano et le cartésianisme »), il est plus que probable que l'auteur de La Mort d'Agrippine se soit servi du philosophème du genius malignus comme fond caché de sa tragédie. On pourrait également se souvenir de la polémique du « Jeune Homme » dans L'Autre Monde cyranien qui attaque la conception que le XVII ${ }^{\mathrm{e}}$ siècle s'était faite de Dieu comme un « Dieu ou sot ou malicieux » (S. Cyrano de Bergerac, Voyage dans la Lune, M. Laugaa (dir.), Garnier-Flammarion, Paris 1970, p. 116 [je souligne]).

${ }^{34}$ C'est la raison avancée par E. Harth qui empêche l'identification simpliste de Séjanus avec le véritable « scélérat» de la pièce : «a villain must [...] be contrasted with goodness, and one would have to conduct a careful search to find it in this play » (op. cit., p. 29). 
amère face à l'optimisme politique d'un Pierre Corneille, si l'on compare La Mort d'Agrippine avec une pièce plus ou moins contemporaine comme Cinna (1642): rien n'est plus loin d'un Tibère que la clémence d'Auguste ${ }^{35}$. Lâche et sans pitié, l'empereur, chez Cyrano, poursuit ses adversaires politiques jusqu'à leur mort atroce. Certes, Séjanus montre « une certaine grandeur » ${ }^{36}$ comme « soldat philosophe » (MA, v. 1650), comme libertin courageux ayant lu Lucrèce, mais il n'est pas moins cruel que l'empereur dont il aspire à arracher violemment le trône.

Et Agrippine, l'héroïne titulaire ? Sa vengeance est avant tout une obsession pathologique, bien que ce motif lui donne aussi une grandeur théâtrale et monstrueuse, tout comme Séjanus se voit ennobli jusqu'à un certain point par son courage et peut-être aussi par la philosophie qu'il professe. Agrippine est cependant aussi abjecte que lui pour la majorité du public et pour les autorités. Aucun des protagonistes de la pièce ne peut donc entrer dans la catégorie aristotélicienne du " héros moyen », tous sont au fond des monstres. Tout comme les personnages de Cinna ont finalement le cœur pur et noble, de sorte que la clémence d'Auguste procède d'une conséquence immanente à la pièce, il est également conséquent, selon la logique interne de la tragédie de Cyrano, que tous périssent dans la catastrophe ou se voient au moins jugés coupables aux yeux du public. Alors qu'il n'y a pas de doute possible sur la véracité de la clémence d'Auguste chez Corneille, tout s'avère mensonge et tromperie dans La Mort d'Agrippine : " tout est faux ${ }^{37}$, voilà comment un critique de la pièce en résume le fond et la forme.

Le scandale provoqué par la tragédie de Cyrano de Bergerac se dégage donc principalement des propos libertins, politiques et philosophiques prononcés sur scène. Dans La Mort d'Agrippine, il devient au moins concevable (et prononçable !) que Dieu n'existe pas et que son représentant sur terre, le monarque, soit un monstre politique, entouré d'une cour regroupant des conspirateurs aussi criminels et monstrueux que lui-même. Il n'est pas surprenant que cette vision politique d'un «monde où règne une corruption universelle » ${ }^{38}$ fût intolérable pour les autorités contemporaines, tant monarchiques qu'ecclésiastiques. L'interdiction

35 Voir C.J. Gossip, op. cit., p. XII : « Comme il [Tibère] est loin [...] de la générosité d'un héros cornélien ! ", et (très laconique) J. Prévot, "Tibère anti-Auguste », [dans :] idem, Cyrano de Bergerac. Poète et Dramaturge, Belin, Paris 1978, p. 199. Sur la surface, La Mort d'Agrippine montre néanmoins quelques parallèles avec le Cinna de Pierre Corneille ( $c f$. S. Cyrano de Bergerac, Euvres complètes, t. 3, p. 293, note 135, et P. Corneille, Cinna, G. Forestier (dir.), Gallimard, Paris 1994, vv. 651-664). À comparer avec les remarques de P.-A. Brun (Savinien de Cyrano Bergerac. Sa vie et ses ouvres d'après des documents inédits, Armand Colin, Paris 1893, p. 235 sq). En ce qui concerne le message politique, les deux pièces ne pourraient pourtant pas diverger plus.

36 J.-Ch. Darmon, Le songe libertin..., p. 39. Voir aussi C.J. Gossip, op. cit., p. XV, et H. Mazaheri pour qui Séjanus est « le seul libre penseur de la pièce» (voir idem, op. cit., p. 61 : « Le plus courageux [des protagonistes] se trouve être le matérialiste, l'athée, l'homme du peuple, le "philosophe" de la pièce : Séjanus »).

37 D. Moncond'huy, op. cit., p. 15.

38 J.-Ch. Darmon, Le songe libertin..., p. 40. 
de la seule tragédie de Cyrano découle par conséquent du scandale inhérent à cette pièce politique extrêmement atypique pour le Grand Siècle.

\author{
POLITICS ACCORDING TO CYRANO DE BERGERAC'S \\ LA MORT D'AGRIPPINE [THE DEATH OF AGRIPPINA]: \\ CONSPIRACY, ATHEISM, AND LIBERTINAGE ÉRUDIT ON STAGE
}

\begin{abstract}
Summary
The present article examines the vision of politics, especially linked to religion, in the only known tragedy by French author Cyrano de Bergerac. Provoking a major scandal during its premiere due to several dialogues considered as atheist by contemporary authorities, this complex piece of dramatic art provides some insights into the political thought of a $17^{\text {th }}$-century philosophical (underground) tendency known as libertinage érudit. As I try to show, one of the major problems we face in the tragedy's (political) interpretation is its overall composition, which Cyrano based on baroque principles like (dis)simulation, illusion, and manipulation, turning the whole piece into a dramatic trompe-l'œil. Focusing on the close reading of some crucial dialogues that involve three of the tragedy's protagonists (Agrippina, Emperor Tiberius, and Sejanus), this article postulates two axes as central to politics according to Cyrano: (1) religion is useful for political enterprises and the foundation of the state, but has no basis in itself since the gods do not exist - they are mere creations of the human mind. (2) At least in Cyrano's Rome, there simply are no good politicians ('good' understood in a moral sense), only brilliant liars and deceivers, Machiavellian rulers, cruel emperors, and equally cruel conspirators. Politics is perceived as a dirty business where there is no space for morals - scandalous conclusions explaining the immediate suppression of any further representations of La Mort d'Agrippine after its tumultuous premiere.
\end{abstract}

Key words: Cyrano de Bergerac, La Mort d'Agrippine, libertinage, political thought, atheism, tragedy, rhetoric, baroque. 